\section{Библиографический список}

1. Абросимов М. Б. Графовые модели отказоустойчивости. Саратов : Изд-во Сарат. ун-та, 2012. 192 с.

2. Hayes J. P. A graph model for fault-tolerant computing system // IEEE Trans. Comput. 1976. Vol. C.25, № 9. P. $875-884$.

3. Абросимов М. Б. О сложности некоторых задач, связанных с расширениями графов // Мат. заметки. 2010.

Т. 88, № 5. С. 643-650.
4. Абросимов М. Б. Характеризация графов с заданным числом дополнительных ребер минимального вершинного 1-расширения // Прикладная дискретная математика. 2012. № 1. С. 111-120.

5. Абросимов М. Б. Минимальные вершинные расширения направленных звезд // Дискретная математика. 2011. Т. 23, № 2. С. 93-102. DOI: $10.4213 / \mathrm{dm} 1144$.

\title{
Characterization of Graphs with a Small Number of Additional Arcs in a Minimal 1-vertex Extension
}

\section{B. Abrosimov, O. V. Modenova}

Saratov State University, Russia, 410012, Saratov, Astrahanskaya st., 83, mic@ rambler.ru, oginiel@ rambler.ru

A graph $G^{*}$ is a $k$-vertex extension of a graph $G$ if every graph obtained from $G^{*}$ by removing any $k$ vertices contains $G$. $k$-vertex extension of a graph $G$ with $n+k$ vertices is called minimal if among all $k$-vertex extensions of $G$ with $n+k$ vertices it has the minimal possible number of arcs. We study directed graphs, whose minimal vertex 1-extensions have a specific number of additional arcs. A solution is given when the number of additional arcs equals one or two.

Key words: minimal vertex extension, exact extension, fault tolerance, graph theory.

\section{References}

1. Abrosimov M. B. Grafouye modeli otkazoustoichivosti [Graph models of fault tolerance]. Saratov, Saratov Univ. Press, 2012, 192 p. (in Russian).

2. Hayes J. P. A graph model for fault-tolerant computing system. IEEE Trans. Comput., 1976, vol. C.25, no. 9, pp. 875-884.

3. Abrosimov M. B. On the Complexity of Some Problems Related to Graph Extensions. Math. Notes, 2010, vol. 88, no. 5, pp. 619-625.

4. Abrosimov M. B. Characterization of graphs with a given number of additional edges in a minimal 1-vertex extension. Prikladnaya Diskretnaya Matematika [Applied Discrete Mathematics], 2012, no. 1, pp. 111-120 (in Russian).

5. Abrosimov M. B. Minimal vertex extensions of directed stars. Diskr. Mat., 2011, vol. 23, no. 2, pp. 93-102 (in Russian). DOI: 10.4213/dm1144.

УДК 629.78

\section{К ТЕОРИИ УСТОЙЧИВОСТИ АВТОНОМНОЙ СИСТЕМЫ УГЛОВОЙ СТАБИЛИЗАЦИИ РЕАКТИВНОГО СНАРЯДА ЗАЛПОВОГО ОГНЯ}

\author{
Д. К. Андрейченко ${ }^{1}$, К. П. Андрейченко ${ }^{2}$, В. В. Кононов ${ }^{3}$ \\ ${ }^{1}$ Доктор фризико-математических наук, зав. кафредрой математического обеспечения вычислительных комплексов и ин- \\ срормационных систем, Саратовский государственный университет им. Н. Г. Чернышевского, AndreichenkoDK@ info.sgu.ru \\ ${ }^{2}$ Доктор технических наук, профрессор кафредры прикладной математики и системного анализа, Саратовский государствен- \\ ный технический университет, kp_andreichenko@ renet.ru \\ ${ }^{3}$ Ассистент кафредры математического обеспечения вычислительных комплексов и инсрормационных систем, Саратовский \\ государственный университет им. Н. Г. Чернышевского, valentin.kononov@ gmail.com
}

Проведено исследование влияния продольного ускорения на устойчивость дискретно-континуальной модели одноканальной системы угловой стабилизации с запаздывающим аргументом упругого вращающегося стержня. Развиты методы построения областей асимптотической устойчивости и анализа импульсных переходных фуункций рассматриваемой комбинированной динамической системы, уравнения движения которой могут быть проанализированы лишь на основе численных методов либо методов асимптотического интегрирования. Определены критические значения продольного ускорения.

Ключевые слова: комбинированные динамические системы, системы стабилизации. 


\section{ВВЕДЕНИЕ}

В работе [1] сформирована комбинированная динамическая модель реактивного снаряда в виде вращающегося вдоль продольной оси упруговязкого стержня, на концах которого закреплены абсолютно жесткие тела реактивного двигателя и головной части снаряда. При этом удалось развить известные методы [2] теории газореактивных систем стабилизации упругодеформируемых конструкций применительно к реактивным снарядам залпового огня. Применительно к снарядам с дальностью стрельбы до 200 км и более весьма актуальными становятся исследование влияния продольного ускорения на области асимптотической устойчивости системы стабилизации, а также определение критических значений продольного ускорения. Но данная математическая модель может быть проанализирована лишь на основе численных методов [3] либо на основе методов асимптотического интегрирования [4].

\section{1. МОДЕЛЬНЫЕ УРАВНЕНИЯ КОМБИНИРОВАННОЙ ДИНАМИЧЕСКОЙ СИСТЕМЫ}

После приведения к безразмерным переменным и параметрам [1] в пренебрежении величинами высшего порядка малости модельные уравнения автономной системы стабилизации упругодеформируемого реактивного снаряда залпового огня принимает вид

$$
\begin{gathered}
\tau_{0} \dot{\varphi}+\varphi=-\tau_{0}\left(\dot{\varphi}_{1}+\dot{\varphi}_{2}\right), \quad m_{1} \ddot{w}_{0}+b \varphi_{1}=N_{1}+P_{1}, \\
J_{1} \ddot{\varphi}_{1}=L_{1}-\xi_{1} N_{1}, \quad J_{2}\left(\ddot{\varphi}_{1}+\ddot{\varphi}_{2}\right)=L_{2}+\xi_{2} N_{2}, \\
m_{2}\left[\ddot{w}_{0}+\ddot{w}_{1}-\left(1+\xi_{1}+\xi_{2}\right) \ddot{\varphi}_{1}\right]=N_{2}-n e^{i(\Omega t-\theta)} \varphi(t-\tau)-m_{2} a_{z} \varphi_{2}, \\
\ddot{u}+(1-i \gamma \Omega) u^{\prime \prime \prime \prime}+\gamma \dot{u}^{\prime \prime \prime \prime}+a_{z}\left[\left(m_{2}+1-z\right) u^{\prime \prime}-u^{\prime}\right]=-\ddot{w}_{0}+\left(z+\xi_{1}\right) \ddot{\varphi}_{1}, \\
u(0, t)=0, \quad u^{\prime}(0, t)=0, \quad u(1, t)=w_{1}(t), \quad u^{\prime}(1, t)=-\varphi_{2}(t), \\
N_{1}=-(1-i \gamma \Omega) u^{\prime \prime \prime}(0, t)-\gamma \dot{u}^{\prime \prime \prime}(0, t), \quad N_{2}=(1-i \gamma \Omega) u^{\prime \prime \prime}(1, t)+\gamma \dot{u}^{\prime \prime \prime}(1, t), \\
L_{1}=-(1-i \gamma \Omega) u^{\prime \prime}(0, t)-\gamma \dot{u}^{\prime \prime}(0, t), \quad L_{2}=(1-i \gamma \Omega) u^{\prime \prime}(1, t)+\gamma \dot{u}^{\prime \prime}(1, t), \\
\varphi(0)=\varphi_{i}(0)=\dot{\varphi}_{i}(0)=0, \quad i=1,2, \\
w_{0}(0)=w_{1}(0)=\dot{w}_{0}(0)=\dot{w}_{1}(0)=0, \quad u(z, 0)=\dot{u}(z, 0)=0 .
\end{gathered}
$$

Здесь

$$
\begin{aligned}
& \varphi(t)=-\beta(t)+i \alpha(t), \quad u(z, t)=x(z, t)+i y(z, t), \quad w_{j}(t)=x_{j}(t)+i y_{j}(t), \quad \varphi_{j}(t)=-\beta_{j}(t)+i \alpha_{j}(t), \\
& N_{j}(t)=N_{x_{j}}(t)+i N_{y_{j}}(t), \quad L_{j}(t)=-L_{y_{j}}(t)+i L_{x_{j}}(t), \quad j=1,2, \quad P_{1}(t)=P_{x_{1}}(t)+i P_{y_{1}}(t), \quad(3)
\end{aligned}
$$

$z$ - индивидуальная пространственная координата; $\alpha_{0}, \beta_{0}-$ углы, определяющие направление пуска, вращающегося с угловой скоростью $\Omega$ вокруг продольной оси снаряда; $\alpha_{1}, \beta_{1}$ и $\alpha_{2}, \beta_{2}-$ углы Эйлера-Крылова отклонения оси $z_{1}$ от $z_{0}$ и оси $z_{2}$ от $z_{1} ; \alpha, \beta-$ углы, задающие положение вектора кинетического момента ротора гироскопа; $\left(x_{0}, y_{0}, z_{0}\right)-$ координаты точки $O_{1}$ в системе $O_{0} x_{0} y_{0} z_{0}$; $(x, y, z)$ - координаты точек срединной линии стержня; $\left(x_{1}, y_{1}, 1\right)-$ координаты конечной точки $O_{\ell}$ срединной линии; $N_{x_{j}}, N_{y_{j}}$ и $L_{x_{j}}, L_{y_{j}}(j=1,2)$ - соответственно силы и моменты сил реакций стержня, действующие на тела 1 и $2 ; P_{x_{1}}, P_{y_{1}}$ - поперечные возмущающие силы; $\tau_{0}-$ постоянная времени гироскопа; $\theta-$ угол опережения установки газореактивных исполнительных двигателей относительно осей выходных сигналов гироскопического датчика угловых перемещений; $\tau-$ время запаздывания газореактивных исполнительных двигателей; $n-$ коэффициент обратной связи; $a_{z}-$ безразмерное продольное ускорение; $m_{j}, J_{j}(j=1,2)-$ соответственно масса и экваториальный момент инерции абсолютно жестких тел 1 и $2 ;(\cdot)^{\prime}=\partial(\cdot) / \partial z ;$ точкой сверху обозначено дифференцирование по времени $t$. Уравнения (1) образуют комбинированную [5] динамическую систему (КДС), содержащую обыкновенные дифференциальные уравнения, уравнения в частных производных, граничные условия, условия связи и начальные условия. При $a_{z}<1$ уравнения (2) переходят в аналогичные уравнения из [1]. Набор величин $\mathbf{x}(t)=\left(P_{x_{1}}(t), P_{y_{1}}(t)\right)^{T}$ рассматривается как входная вектор-функция, а набор величин $\mathbf{y}(t)=\left(\alpha_{1}(t), \beta_{1}(t), x_{0}(t), y_{0}(t)\right)^{T}$ представляет собой выходную вектор-функцию КДС. Поскольку система уравнений (2) линейна, в ней выполняется одностороннее 
интегральное преобразование Лапласа $\tilde{f}(\lambda)=L[f(t)]=\int_{0}^{\infty} f(t) e^{-\lambda t} d t$ и далее исключается величина $\tilde{\varphi}(\lambda)$ (символ « » над изображением Лапласа далее опущен). Поскольку аналог краевая задачи (2) в изображениях Лапласа линейным образом зависит от величин $\lambda^{2} w_{0}(\lambda), w_{1}(\lambda), \lambda^{2} \varphi_{1}(\lambda), \varphi_{2}(\lambda)$, то

$$
\begin{gathered}
N_{k}(\lambda)=[1+\gamma(\lambda-i \Omega)]\left[N_{k}^{(1)}(\lambda) \lambda^{2} w_{0}(\lambda)+N_{k}^{(2)}(\lambda) w_{1}(\lambda)+N_{k}^{(3)}(\lambda) \lambda^{2} \varphi_{1}(\lambda)+N_{k}^{(4)}(\lambda) \varphi_{2}(\lambda)\right], \quad \\
L_{k}(\lambda)=[1+\gamma(\lambda-i \Omega)]\left[L_{k}^{(1)}(\lambda) \lambda^{2} w_{0}(\lambda)+L_{k}^{(2)}(\lambda) w_{1}(\lambda)+L_{k}^{(3)}(\lambda) \lambda^{2} \varphi_{1}(\lambda)+L_{k}^{(4)}(\lambda) \varphi_{2}(\lambda)\right], \quad k=1,2 .
\end{gathered}
$$

Входящие в выражения (4) функции $N_{k}^{(j)}(\lambda), L_{k}^{(j)}(\lambda), k=1,2, j=1,2,3,4$, являются решением следующих вспомогательных линейных краевых задач:

$$
\begin{gathered}
\lambda^{2} u(z, \lambda)+[1+\gamma(\lambda-i \Omega)] u^{\prime \prime \prime \prime}(z, \lambda)+a_{z}\left[\left(m_{2}+1-z\right) u^{\prime \prime}(z, \lambda)-u^{\prime}(z, \lambda)\right]=-\delta_{j}^{1}+\delta_{j}^{3}\left(z+\xi_{1}\right), \\
u(0, \lambda)=0, \quad u^{\prime}(0, \lambda)=0, \quad u(1, \lambda)=\delta_{j}^{2}, \quad u^{\prime}(1, \lambda)=-\delta_{j}^{4}, \\
N_{1}^{(j)}(\lambda)=-u^{\prime \prime \prime}(0, \lambda), \quad N_{2}^{(j)}(\lambda)=u^{\prime \prime \prime}(1, \lambda), \quad L_{1}^{(j)}(\lambda)=-u^{\prime \prime}(0, \lambda) \\
L_{2}^{(j)}(\lambda)=u^{\prime \prime}(1, \lambda), \quad j=1,2,3,4, \quad \delta_{j}^{\nu}-\text { символ Кронекера. }
\end{gathered}
$$

Аналогично [1] из (1) в изображениях Лапласа и (4) следуют выражения для передаточных функций системы стабилизации по угловой ошибке $\varphi_{1}$ и поперечному смещению $w_{0}$

$$
\begin{aligned}
& \Pi(\lambda)=\frac{\Delta_{\varphi_{1}}(\lambda)}{\Delta(\lambda)}, \quad \Pi^{0}(\lambda)=\frac{\Delta_{w_{0}}(\lambda)}{\lambda \Delta(\lambda)}, \quad \varphi_{1}(\lambda)=\Pi(\lambda) P_{1}(\lambda), \quad w_{0}(\lambda)=\Pi^{0}(\lambda) P_{1}(\lambda), \\
& \Delta(\lambda)=\left|\begin{array}{cccc}
\psi_{11}(\lambda) & \psi_{12}(\lambda) & \lambda \psi_{13}(\lambda) & \psi_{14}(\lambda) \\
\psi_{21}(\lambda) & \psi_{22}(\lambda) & \psi_{23}(\lambda) & \psi_{24}(\lambda) \\
\psi_{31}(\lambda) & \psi_{32}(\lambda) & \lambda \psi_{33}(\lambda) & \psi_{34}(\lambda) \\
\psi_{41}(\lambda) & \psi_{42}(\lambda) & \lambda \psi_{43}(\lambda) & \psi_{44}(\lambda)
\end{array}\right| \\
& \Delta_{\varphi_{1}}(\lambda)=-\operatorname{det}\left[\psi_{\nu j}(\lambda)\right], \quad \nu=1,3,4, \quad j=1,2,4, \\
& \Delta_{w_{0}}(\lambda)=-\operatorname{det}\left[\psi_{\nu j}(\lambda)\right], \quad \nu=1,3,4, \quad j=2,3,4 \\
& \psi_{11}(\lambda)=[1+\gamma(\lambda-i \Omega)]\left[\xi_{1} N_{1}^{(1)}(\lambda)-L_{1}^{(1)}(\lambda)\right], \quad \psi_{12}(\lambda)=[1+\gamma(\lambda-i \Omega)]\left[\xi_{1} N_{1}^{(2)}(\lambda)-L_{1}^{(2)}(\lambda)\right], \\
& \psi_{13}(\lambda)=\lambda\left[J_{1}+(1+\gamma(\lambda-i \Omega))\left(\xi_{1} N_{1}^{(3)}(\lambda)-L_{1}^{(3)}(\lambda)\right)\right], \quad \psi_{14}(\lambda)=[1+\gamma(\lambda-i \Omega)]\left[\xi_{1} N_{1}^{(4)}(\lambda)-L_{1}^{(4)}(\lambda)\right], \\
& \psi_{21}(\lambda)=m_{1}-[1+\gamma(\lambda-i \Omega)] N_{1}^{(1)}(\lambda), \quad \psi_{22}(\lambda)=-[1+\gamma(\lambda-i \Omega)] N_{1}^{(2)}(\lambda), \\
& \psi_{23}(\lambda)=b-[1+\gamma(\lambda-i \Omega)] \lambda^{2} N_{1}^{(3)}(\lambda), \quad \psi_{24}(\lambda)=-[1+\gamma(\lambda-i \Omega)] N_{1}^{(4)}(\lambda), \\
& \psi_{31}(\lambda)=-[1+\gamma(\lambda-i \Omega)]\left[\xi_{2} N_{2}^{(1)}(\lambda)+L_{2}^{(1)}(\lambda)\right], \quad \psi_{32}(\lambda)=-[1+\gamma(\lambda-i \Omega)]\left[\xi_{2} N_{2}^{(2)}(\lambda)+L_{2}^{(2)}(\lambda)\right], \\
& \psi_{33}(\lambda)=\lambda\left[J_{2}-(1+\gamma(\lambda-i \Omega))\left(\xi_{2} N_{2}^{(3)}(\lambda)+L_{2}^{(3)}(\lambda)\right)\right], \\
& \psi_{34}(\lambda)=J_{2} \lambda^{2}-[1+\gamma(\lambda-i \Omega)]\left[\xi_{2} N_{2}^{(4)}(\lambda)+L_{2}^{(4)}(\lambda)\right], \\
& \psi_{41}(\lambda)=\left(1+\tau_{0} \lambda\right)\left(m_{2}-[1+\gamma(\lambda-i \Omega)] N_{2}^{(1)}(\lambda)\right), \\
& \psi_{42}(\lambda)=\left(1+\tau_{0} \lambda\right)\left(m_{2} \lambda^{2}-[1+\gamma(\lambda-i \Omega)] N_{2}^{(2)}(\lambda)\right), \\
& \psi_{43}(\lambda)=-m_{2}\left(1+\xi_{1}+\xi_{2}\right) \lambda\left(1+\tau_{0} \lambda\right)-n \tau_{0} e^{-i \theta-\tau(\lambda-i \Omega)}-[1+\gamma(\lambda-i \Omega)] \lambda N_{2}^{(3)}(\lambda)\left(1+\tau_{0} \lambda\right), \\
& \psi_{44}(\lambda)=m_{2} a_{z}\left(1+\tau_{0} \lambda\right)-n \tau_{0} e^{-i \theta-\tau(\lambda-i \Omega)}-\left(1+\tau_{0} \lambda\right)[1+\gamma(\lambda-i \Omega)] N_{2}^{(4)}(\lambda) .
\end{aligned}
$$

Компоненты изображений Лапласа входной $\mathbf{x}(\lambda)=\left(P_{x_{1}}(\lambda), P_{y_{1}}(\lambda)\right)^{T}$ и выходной $\mathbf{y}(\lambda)=\left(\alpha_{1}(\lambda)\right.$, $\left.\beta_{1}(\lambda), x_{0}(\lambda), y_{0}(\lambda)\right)^{T}$ вектор-функций связаны следующим образом:

$$
\begin{aligned}
& {\left[\begin{array}{c}
-\beta_{1}(\lambda) \\
\alpha_{1}(\lambda)
\end{array}\right]=\left[\begin{array}{cc}
\Pi_{11}(\lambda) & \Pi_{12}(\lambda) \\
\Pi_{21}(\lambda) & \Pi_{22}(\lambda)
\end{array}\right]\left[\begin{array}{c}
P_{x_{1}}(\lambda) \\
P_{y_{1}}(\lambda)
\end{array}\right], \quad\left[\begin{array}{c}
x_{0}(\lambda) \\
y_{0}(\lambda)
\end{array}\right]=\left[\begin{array}{cc}
\Pi_{11}^{0}(\lambda) & \Pi_{12}^{0}(\lambda) \\
\Pi_{21}^{0}(\lambda) & \Pi_{22}^{0}(\lambda)
\end{array}\right]\left[\begin{array}{c}
P_{x_{1}}(\lambda) \\
P_{y_{1}}(\lambda)
\end{array}\right],} \\
& \Pi_{11}(\lambda)=\Pi_{22}(\lambda)=[\Pi(\lambda)+\overline{\Pi(\bar{\lambda})}] / 2=Q_{1}(\lambda) / D(\lambda), \\
& \Pi_{12}(\lambda)=-\Pi_{21}(\lambda)=i[\Pi(\lambda)-\overline{\Pi(\bar{\lambda})}] / 2=Q_{2}(\lambda) / D(\lambda), \\
& \Pi_{11}^{0}(\lambda)=\Pi_{22}^{0}(\lambda)=\left[\Pi^{0}(\lambda)+\overline{\Pi^{0}(\bar{\lambda})}\right] / 2=Q_{1}^{0}(\lambda) / \lambda D(\lambda), \\
& \Pi_{12}^{0}(\lambda)=-\Pi_{21}^{0}(\lambda)=i\left[\Pi^{0}(\lambda)-\overline{\Pi^{0}(\bar{\lambda})}\right] / 2=Q_{2}^{0}(\lambda) / \lambda D(\lambda),
\end{aligned}
$$


причем характеристический и возмущающие квазимногочлены суть

$$
\begin{gathered}
D(\lambda)=\Delta(\lambda) \overline{\Delta(\bar{\lambda})}, \quad Q_{1}(\lambda)=\left[\Delta_{\varphi_{1}}(\lambda) \overline{\Delta(\bar{\lambda})}+\overline{\Delta_{\varphi_{1}}(\bar{\lambda})} \Delta(\lambda)\right] / 2, \\
Q_{2}(\lambda)=i\left[\Delta_{\varphi_{1}}(\lambda) \overline{\Delta(\bar{\lambda})}-\overline{\Delta_{\varphi_{1}}(\bar{\lambda})} \Delta(\lambda)\right] / 2, \quad Q_{1}^{0}(\lambda)=\left[\Delta_{w_{0}}(\lambda) \overline{\Delta(\bar{\lambda})}+\overline{\Delta_{w_{0}}(\bar{\lambda})} \Delta(\lambda)\right] / 2, \\
Q_{2}^{0}(\lambda)=i\left[\Delta_{w_{0}}(\lambda) \overline{\Delta(\bar{\lambda})}-\overline{\Delta_{w_{0}}(\bar{\lambda})} \Delta(\lambda)\right] / 2, \\
D(\bar{\lambda})=\overline{D(\lambda)}, \quad Q_{\nu}(\bar{\lambda})=\overline{Q_{\nu}(\lambda)}, \quad Q_{\nu}^{0}(\bar{\lambda})=\overline{Q_{\nu}^{0}(\lambda)}, \quad \nu=1,2 .
\end{gathered}
$$

\section{2. РЕШЕНИЕ МОДЕЛЬНЫХ КРАЕВЫХ ЗАДАЧ}

При умеренных и средних значениях $|\lambda|$ выполнялось численное решение краевой задачи (5) на основе проекционного метода Галеркина в предположении

$$
u(z, \lambda) \approx \sum_{k=0}^{N+4} u_{k} T_{k}(2 z-1), \quad 0 \leqslant z \leqslant 1 .
$$

Здесь $T_{k}(z)=\cos (k \arccos z)$ - ортогональные полиномы Чебышева 1-го рода. Краевая задача (5) сводилась к дискретному аналогу - системе линейных уравнений относительно Фурье-коэффициентов $u_{k}, k=0,1, \ldots, N_{u}+4$. Выражения для вспомогательных функций $N_{k}^{(j)}(\lambda), L_{k}^{(j)}(\lambda), k=1,2$, $j=1,2,3,4$, не имеют особенностей в конечной подобласти правой комплексной полуплоскости $(\lambda)$, если там нет нулей определителя дискретного аналога (5), что достаточно просто проверяется на основе принципа аргумента. Во всех рассмотренных ниже случаях вспомогательные функции $N_{k}^{(j)}(\lambda)$, $L_{k}^{(j)}(\lambda), k=1,2, j=1,2,3,4$, не имели особенностей в правой комплексной полуплоскости $(\lambda)$. Приближенное вычисление вспомогательных функций $N_{1}^{(j)}(\lambda), N_{2}^{(j)}(\lambda), L_{1}^{(j)}(\lambda), L_{2}^{(j)}(\lambda), j=1,2,3,4$, при $|\lambda|>1, \operatorname{Re} \lambda \geqslant-\sigma_{0}, 0<\sigma_{0}<\infty$, удобно выполнять на основе асимптотического интегрирования вспомогательных линейных краевых задач (5) по методу Вентцеля-Крамерса-Бриллюэна (ВКБ) [4]. Частные решения однородного уравнения (5) представляются в виде $e^{ \pm k V(z, \pm k)}, e^{ \pm i k V(z, \pm i k)}$, где $k=k(\lambda)=\left[-\lambda^{2} /(1+\gamma(\lambda-i \Omega))\right]^{1 / 4}$, и используется асимптотическое разложение:

$$
V(z, k)=V_{0}(z)+k^{-1} V_{1}(z)+k^{-2} V_{2}(z)+k^{-3} V_{3}(z)+\ldots
$$

В результате при $|\lambda|>1, \operatorname{Re} \lambda \geqslant-\sigma_{0}, 0<\sigma_{0}<\infty$ функции $N_{k}^{(j)}(\lambda), L_{k}^{(j)}(\lambda), k=1,2, j=1,2,3,4$, являются аналитическими функциями $\lambda$, причем

$$
\begin{gathered}
\lim _{\lambda \rightarrow \infty} \lambda^{-14} D(\lambda)=\left(m_{1} m_{2} \tau_{0} J_{1} J_{2}\right)^{2}, \quad \lim _{\lambda \rightarrow \infty} \lambda^{-47 / 4} Q_{1}(\lambda)=\sqrt{2} \xi_{1} \gamma^{1 / 4} m_{1} \tau_{0} J_{1}\left(m_{2} J_{2}\right)^{2}, \\
\lim _{\lambda \rightarrow \infty} \lambda^{-13} Q_{1}^{0}(\lambda)=\tau_{0} m_{1}\left(m_{2} J_{1} J_{2}\right)^{2}, \quad \operatorname{Re} \lambda>-\infty,
\end{gathered}
$$

а обобщенные степени возмущающих квазимногочленов $Q_{2}(\lambda)$ и $Q_{2}^{0}(\lambda)$ характеризуются величинами, меньшими 47/4 и 13 соответственно.

\section{3. МОДЕЛИРОВАНИЕ УСТОЙЧИВОСТИ КОМБИНИРОВАННОЙ ДИНАМИЧЕСКОЙ СИСТЕМЫ}

Из теорем об устойчивости КДС [5] следует, что система стабилизации будет устойчива при выполнении условия

$$
\underset{0 \leqslant \omega<\infty}{\Delta} \arg D(i \omega)=7 \pi
$$

и неустойчива - в других случаях. Для управляемых КДС характеристический квазимногочлен является функцией не только комплексного параметра $\lambda$, но и параметров обратных связей

$$
D(\lambda, \theta, n)=\Delta(\lambda, \theta, n) \overline{\Delta(\bar{\lambda}, \theta, n)}
$$

Аналогично [1] параметрические уравнения возможных границ области устойчивости на плоскости параметров $(\theta, n)$ принимают вид

$\operatorname{Re} \Delta(i \omega, \theta, n)=0, \quad \operatorname{Im} \Delta(i \omega, \theta, n)=0, \quad \operatorname{Re} \Delta(-i \omega, \theta, n)=0, \quad \operatorname{Im} \Delta(-i \omega, \theta, n)=0, \quad 0 \leqslant \omega<\infty$ 
Рассмотрим модель системы стабилизации с параметрами $\tau_{0}=7, \gamma=0.06, m_{1}=0.06, m_{2}=0.1$, $J_{1}=0.015, J_{2}=0.02, \xi_{1}=0.03, \xi_{2}=0.05, \tau=0.07, p_{y_{1}}=0.01, p_{0}=0.01$ при $\Omega=5, b=0.02$ и $\Omega=9$, $b=0.0648$ для значений коэффициента внутреннего трения $\gamma=0.06$ и $\gamma=0.01$.

На рис. 1 представлены границы областей устойчивости для значений перегрузки $a_{z}=0,2,5$, 10 и $\gamma=0.06$. При увеличении значения перегрузки $a_{z}$ область устойчивости постепенно смещается вправо на плоскости параметров $(\theta, n)$.

Аналогичные результаты для границ областей устойчивости для значений коэффициента внутреннего трения $\gamma=0.01$ представлены на рис. 2 .

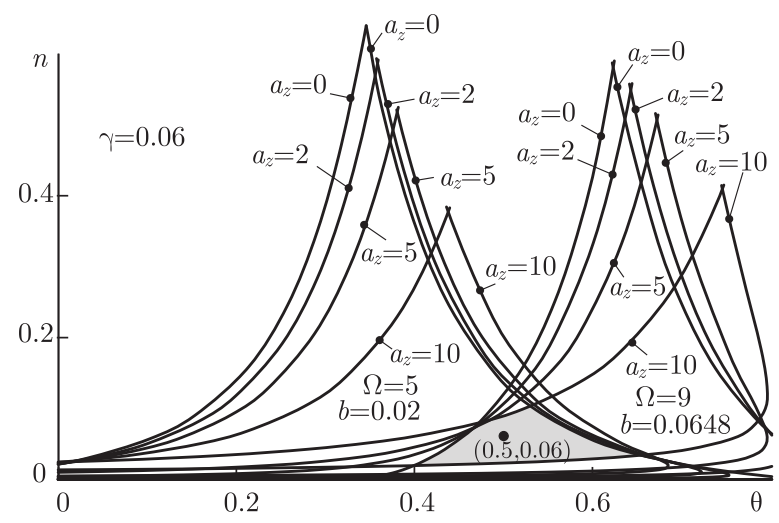

Рис. 1. Область устойчивости модели при $\gamma=0.06$

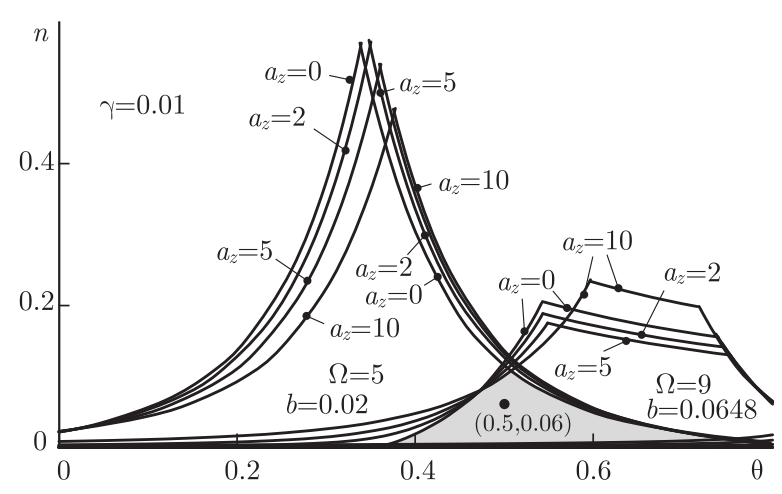

Рис. 2. Область устойчивости модели при $\gamma=0.01$

Как видно, изменение коэффициента внутреннего трения $\gamma$ при возрастании угловой скорости вращения снаряда $\Omega$ значительно сокращает области устойчивости. Для реактивных снарядов залпового огня представляет интерес увеличение массы $m_{2}$ головной части снаряда.

На рис. 3, a, б для системы стабилизации с параметрами $\tau_{0}=7, m_{1}=0.06, J_{1}=0.015, \xi_{1}=0.03$, $\xi_{2}=0.05, \tau=0.07$ при $\Omega=5,7,8,9$ и $b=0.02(\Omega / 5)^{2}$ для значений коэффициента внутреннего трения $\gamma=0.06$ и $\gamma=0.01$ приведены графики критических перегрузок $a_{z}$ в зависимости от массы головной части снаряда $m_{2}$ (при пропорциональном изменении момента инерции головной части снаряда $J_{2}=0.2 m_{2}$ ). Критические значения перегрузок $a_{z}$, при которых происходит потеря устойчивости системы стабилизации, находились методом бисекции с оптимизацией интервала поиска. Как следует из представленных на рис. 3 результатов, увеличение массы $m_{2}$ головной части снаряда приводит к значительному ограничению на величину угловой скорости $\Omega$ вращения снаряда.
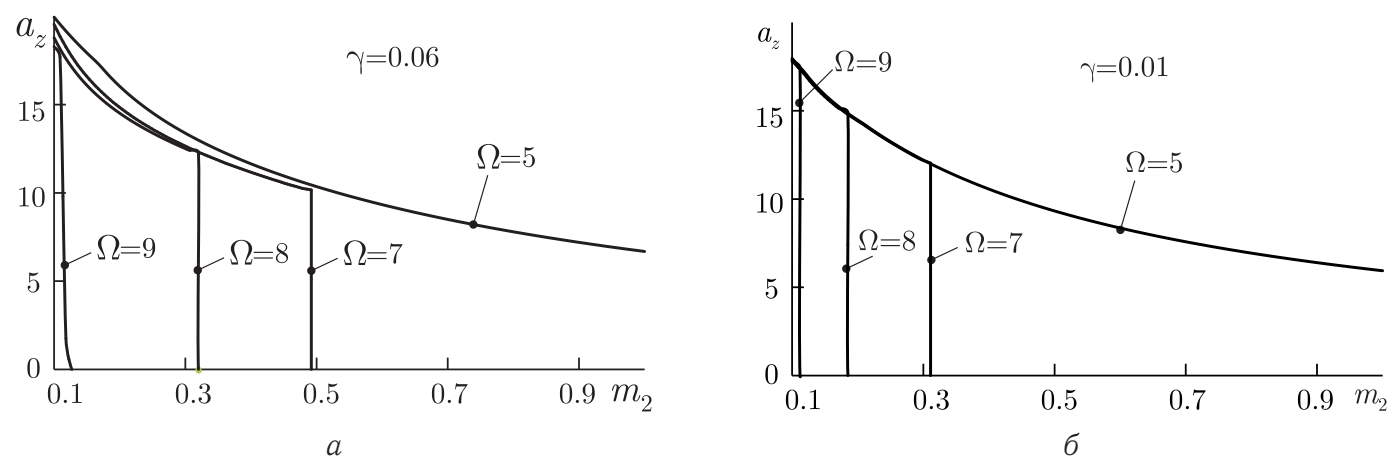

Рис. 3. Значения критических перегрузок модели при $\gamma=0.06$ (a) и $\gamma=0.01$ (б)

\section{Библиографический список}

1. Андрейченко Д. К., Андрейченко К. П. К теории автономных системы угловой стабилизации реактивных снарядов залпового огня // Изв. РАН. Теория и системы управления. 2009. № 3. С. 141-156.
2. Андрейченко Д. К., Андрейченко К. П. К теории стабилизации спутников с упругими стержнями // Изв. РАН. Теория и системы управления. 2004. № 6. С. 150163. 
3. Флетчер К. Численные методы на основе метода Галеркина. М. : Мир, 1988. 352 с.

4. Коул Дж. Методы возмущений в прикладной математике. М. : Мир, 1972. 274 с.
5. Андрейченко Д. К., Андрейченко К. П. К теории комбинированных динамических систем // Изв. РАН. Теория и системы управления. 2000. № 3. С. 54-69.

\title{
On Stability Theory of Autonomous Angular Stabilization System for Combined Dynamical Systems
}

\author{
D. K. Andreichenko ${ }^{1}$, K. P. Andreichenko ${ }^{2}$, V. V. Kononov ${ }^{1}$ \\ ${ }^{1}$ Saratov State University, Russia, 410012, Saratov, Astrahanskaya st., 83, AndreichenkoDK@info.sgu.ru, \\ valentin.kononov@gmail.com \\ ${ }^{2}$ Saratov State Technical University, Russia, 410054, Saratov, Polytechnicheskaya st., 77, kp_andreichenko@ @enet.ru \\ Studied the effect on the stability of the longitudinal acceleration discretely-continuum model of single-channel angular stabilization \\ system with of delayed argument. Methods of construction asymptotic stability areas and analysis of impulse transition functions are \\ developed. The critical values of the longitudinal acceleration are defined.
}

Key words: combined dynamical systems, stabilization systems.

\section{References}

1. Andreichenko D. K., Andreichenko K. P. On the theory of autonomous angular stabilization systems of missiles for salvo firing. J. of Computer and Systems Sciences Intern., 2009, vol. 48, no. 3, pp. 465-480. DOI: 10.1134/S1064230709030137.

2. Andreichenko D. K., Andreichenko K. P. On the theory of stabilization of satellites having elastic rods. J. of Computer and Systems Sciences Intern., 2004, vol. 43, no. 6, pp. 973-986.

3. Fletcher K. Chislennye metody na osnove metoda
Galjorkina [Numerical methods based on the Galerkin method]. Moscow, Mir, 1988, 352 p.

4. Cole J. D. Perturbation methods in applied mathematics. Blaisdell Publishing Co. Ginn and Co., Waltham, Mass.-Toronto, Ont.-London, 1968, 260 p. (Rus. ed.: Cole J. Metody vozmushhenij v prikladnoj matematike. Moscow, Mir, 1972, 274 p.)

5. Andreichenko D. K., Andreichenko K. P. On the theory of hybrid dynamical systems. J. of Computer and Systems Sciences Intern., 2000, vol. 39, no. 3, pp. 383-398.

\section{УДК 519.95}

\section{ИДЕНТИФИКАЦИЯ СТРУКТУРЫ АВТОМАТА ФРАГМЕНТАМИ ПОВЕДЕНИЯ}

\section{С. А. Богомолов}

Кандидат физико-математических наук, доцент касредры прикладной математики и инфрорматики, Саратовский государственный социально-экономический университет, Alexbogomolov@ya.ru

\begin{abstract}
Изучается задача идентификации структуры автомата конечным фррагментом его поведения. Под поведением автомата понимается множество различных о.-д. фрункций, реализуемых в автомате, а под конечным сррагментом поведения -следы о.-д. фуункцй и автоматов. Ведено понятие идентифрицирующего следа для автомата, «неизбыточного» относительно его реализации. Предложен подход, позволяющий в множестве следов, идентисицирующих автомат, выделить и описать конечное множество «неизбыточных» следов, содержащих только необходимую инсрормацию для идентификации автомата.
\end{abstract}

Ключевые слова: автомат, эксперимент с автоматом, подэксперимент эксперимента, след о.-д. фуунции и автомата, идентисрицирующий след автомата, операция редукции следа, неизбыточный идентифицииующий след автомата.

\section{ВВЕДЕНИЕ}

Обширный класс как фундаментальных, так и прикладных проблем анализа дискретных систем допускает их естественное сведение к проблемам идентификации. Л. Льюинг [1] отмечал: «Формирование моделей на основе результатов наблюдений и исследование их свойств - вот по существу основное содержание науки». Модели могут быть более или менее формализованными, но все они 